\title{
Simultaneous determination of aflatoxins, ochratoxin A, and zearalenone in cereals using a validated RP-HPLC method and phred derivatization system
}

\begin{abstract}
The objective of the study was to develop and validate an HPLC-FLD method, after sample preparation and derivatization, for the simultaneous determination of aflatoxins (B1, B2, G1, and G2), ochratoxin A (OTA), and zearalenone (ZEA) in cereals. A solid-liquid extraction approach using $80 \%$ methanol followed by a multifunctional immunoaffinity AOZ column clean-up was applied for the extraction of these mycotoxins before HPLC analysis. A photochemical reactor enhance derivatization system (PHRED) was used between the HPLC column and fluorescence detector, in order to enhance the fluorescence intensity of aflatoxin B1 and G1. The best chromatographic separation for the simultaneous determination of the nominated mycotoxins was achieved under optimal HPLC conditions. Recoveries were 77.31-104.1\% for cereal samples spiked at 6.5, 10, and $100 \mathrm{ng} / \mathrm{g}$ for total aflatoxins (AFs), OTA, and ZEA, respectively; whereas, the precisions were $2.45-11.14 \%$. Limits of detection (LOD) for AFs, OTA, and ZEA were 0.004-0.012, 0.05, and 0.5 ng/g, respectively; whereas. limits of quantification (LOQ) were $0.015-0.05,0.2$, and $2 \mathrm{ng} / \mathrm{g}$, correspondingly.
\end{abstract}

Keyword: Aflatoxins; Cereal; Ochratoxin; RP-HP 